\title{
Neuroprotective Effects of Lacosamide in Experimental Peripheral Nerve Injury in Rats : A Prospective Random- ized and Placebo-Controlled Trial
}

\author{
Serdar Demiroz, ${ }^{1}$ Koray Ur, ${ }^{2}$ Aydin Sukru Bengu, ${ }^{3}$ Aykut Ulucan, ${ }^{3}$ Yunus Atici, ${ }^{4}$ Sinan Erdogan, ${ }^{5}$ Alper Cirakli, ${ }^{6}$ Sevki Erdem \\ Department of Orthopaedics and Traumatology, ${ }^{\dagger}$ Medicalpark Hospital, Kocaeli, Turkey \\ Department of Neurosurgery, ${ }^{2}$ Cigli Regional Education Hospital, Illzmir, Turkey \\ Department of Medical Services and Techniques, ${ }^{3}$ Vocational School of Health Services, Bingol University, Bingöl, Turkey \\ Department of Orthopaedics and Traumatology, ${ }^{4}$ Medicalpark Gebze Hospital, Kocaeli, Turkey \\ Department of Orthopaedics and Traumatology, ${ }^{5}$ Baltalimanı Education and Research Hospital, Istanbul, Turkey \\ Department of Orthopaedics and Traumatology, ${ }^{6}$ Ordu University, Ordu, Turkey \\ Department of Orthopaedics and Traumatology, Medicana Hospital, Istanbul, Turkey
}

Objective : To evaluate the neuroprotective effects of lacosamide after experimental peripheral nerve injury in rats.

Methods : A total of 28 male wistar albino rats weighing 300-350 g were divided into four groups. In group I, the sciatic nerve exposed and the surgical wound was closed without injury; in group II, peripheral nerve injuries (PNI) was performed after dissection of the nerve; in group III, PNI was performed after dissection and lacosamide was administered, and in group IV, PNI was performed after dissection and physiological saline solution was administered. At 7 days after the injury all animals were sacrificed after walking track analysis. A $5 \mathrm{~mL}$ blood sample was drawn for biochemical analysis, and sciatic nerve tissues were removed for histopathological examination.

Results : There is low tissue damage in lacosamide treated group and antioxidant anzymes and malondialdehyde levels were higher than non-treated and placebo treated group. However there was no improvement on clinical assessment.

Conclusion : The biochemical and histological analyses revealed that lacosamide has neuroprotective effect in PNI in rats. This neuroprotective capacity depends on its scavenger role for free oxygen radicals by increasing antioxidant enzyme activity.

Key Words : Lacosamide · Peripheral nerve injuries · Sciatic nerve.

\section{INTRODUCTION}

Peripheral nerve injuries (PNI) can occur due to mechanical, chemical, and thermal reasons. Trauma is the most com- mon reason and it is known that PNI occurs in $2.8 \%$ of trauma patients ${ }^{19}$. Although there are several drugs, steroids and hormones, whose positive effects have been reported clinically and experimentally, recovery after PNI is still a clinical chal-

- Received : August 16, 2019 •Revised : September 10, 2019 •Accepted : September 24, 2019

- Address for reprints : Serdar Demiroz

Department of Orthopaedics and Traumatology, Medicalpark Hospital, Kavaklı street, Gebze, Kocaeli 41400, Turkey

Tel : +90 505 3386490, Fax : +90 42621310 43, E-mail : serdardemiroz@hotmail.com, ORCID : https://orcid.org/0000-0002-2403-3750

This is an Open Access article distributed under the terms of the Creative Commons Attribution Non-Commercial License (http://creativecommons.org/licenses/by-nc/4.0) which permits unrestricted non-commercial use, distribution, and reproduction in any medium, provided the original work is properly cited. 
lenge $e^{2,14,21,24,29)}$. However, unlike in the central nervous system, regeneration in the peripheral nervous system is possible but full functional recovery is often poor ${ }^{9)}$.

It is known that crush in peripheral nervous system results in damage of intraneural microcirculation by direct mechanical injury ${ }^{16,31)}$. An inflammatory response strats and levels of free oxygen radicals increase which leads to further tissue damage. Lipid peroxidation and the level of tissue malondialdehyde (MDA) also increases which is a toxic process after $\mathrm{PNI}^{12)}$.

Therefore, several chemical agents with antioxidant and antiinflammatory effect have been evaluated for their ability to inhibit this cascade ${ }^{3-5,11)}$ Methylprednisolone and gabapentin are the reference agents and have shown beneficial effects in the literature ${ }^{26)}$. But, although they have beneficial effects on parenchymal damage, there is no significant improvement on functional recovery and there are several side effects associated with their use ${ }^{7)}$.

Lacosamide may be another pharmaceutical candidate for treatment after PNI. Although it is an antiepileptic drug in clinical use, it has been shown to protect neurological tissue and have ameliorative effect on peripheral neuropathy. Several studies have reported that it exhibits antioxidant, anti-inflammatory and lipid peroxidation inhibiting effect which are the main scope of the treatment strategy for neural damage after $\mathrm{PNI}^{1,13,22)}$. We have also reported in our previous study that lacosamide has neuroprotective effects after spinal cord injury ${ }^{8)}$. So, when consider the anti-inflammatory, antioxidant, and inhibiting lipid peroxidation properties and protective effects of lacosamide on neural tissue after experimental spinal cord injury, we hypothesized that it may provide neuroprotective effects after traumatic PNI.

To the best of our knowledge, no study has reported on the use of lacosamide in an experimental PNI model. The purpose of this study was to investigate the effects of lacosamide after experimental sciatic nerve crush injury in rats using histopathological, biochemical and clinical methods.

\section{MATERIALS AND METHODS}

All the experimental procedures performed were approved by the Ethics Committee of Bingol University (date and serial number : 12.01.2017-796). A total of 28 male wistar albino rats weighing 300-350 g were divided into four groups of seven rats each. In group I (sham), the sciatic nerve exposed and the surgical wound was closed without injury; in group II, PNI was performed after dissection of the nerve; in group III, PNI was performed after dissection and lacosamide was administered, and, in group IV, PNI was performed after dissection and physiological saline solution was administered.

All rats were kept under environmentally controlled conditions and housed in separate cages during the test and were fed standard rodent food and water. They were anesthetized by intraperitoneal injection of $10 \mathrm{mg} / \mathrm{kg}$ of xylazine (Alfazyne, Egevet, Izmir, Turkey) and $50 \mathrm{mg} / \mathrm{kg}$ of ketamine (Ketalar, Parke-Davis, Eczac1 baş 1, Turkey). The rats were placed in the prone position, and the surgical field cleaned with povidone iodine (Bathicon; Adeka, Samsun, Turkey). A skin incision was made on the left hip of the rats and the sciatic nerve within the gluteal muscle exposed. The surgical wound was closed without PNI in group I. After the same procedure for approach to the sciatic nerve, a vascular clip (Vascu-Statt ${ }^{\circledR}$; Scanlan, St. Paul, MN, USA) with $40 \mathrm{~g}$ closing force was applied to the sciatic nerve for 1 minute which was sufficient to cause mild to severe damage and the crush injury achieved in group II (Fig. 1), and then the wound was closed ${ }^{17)}$. Lacosamide was administered intraperitoneally at a dose of $30 \mathrm{mg} / \mathrm{kg}$

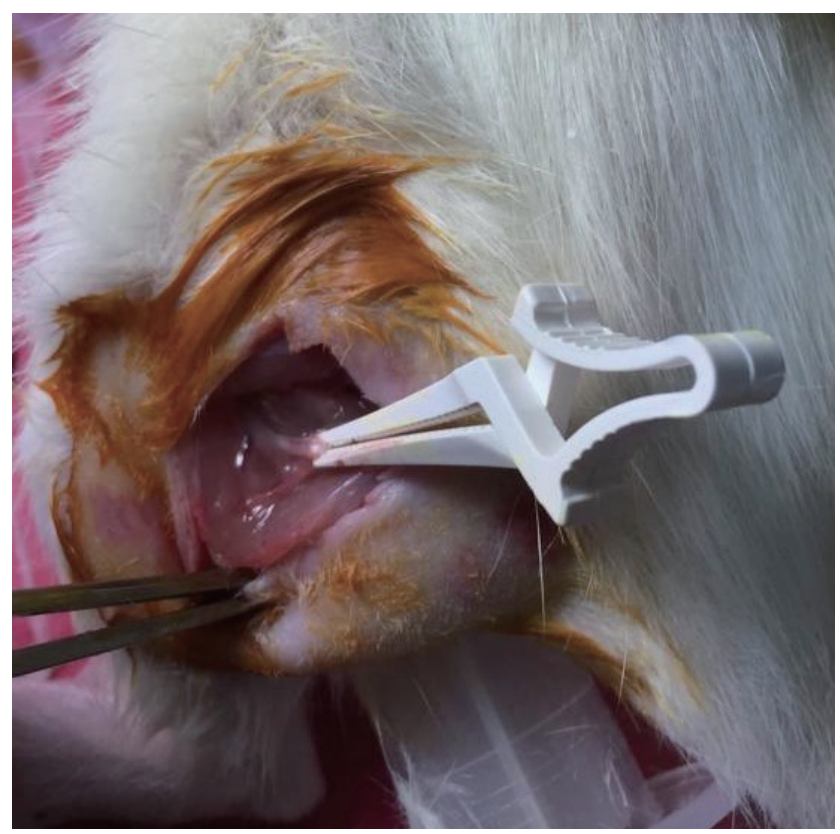

Fig. 1. Compression of the sciatic nerve with a clamp with $40 \mathrm{~g}$ closing force. 
by 8 hours intervals after PNI in group III. The first dose of drug administered immediately after closing the skin and the last dose administered 8 hours before the sacrification and, serum physiologic was administered at the same dose and the same interval in group IV after PNI.

At 7 days after the injury all animals were sacrificed after walking track analysis by decapitation under deep anesthesia and autopsy performed according to the Virchow technique. A $5 \mathrm{~mL}$ blood sample was drawn for biochemical analysis, and sciatic nerve tissues were removed for histopathological examination. Blood samples were centrifuged at $1500 \mathrm{rpm}$ for 15 minutes to obtain serum, and serum samples were stored at $-40^{\circ} \mathrm{C}$ before analysis of the glutathione peroxidase (GPX), superoxide dismutase (SOD), catalase, and MDA levels by using a commercial enzyme-linked immunosorbent assay kit (SunRed, catalog numbers SOD : 201-11-0169, GPX : 201-115104, catalase : 201-11-5106, MDA : 201-11-0157).

The sciatic nerve tissues were fixed in 10\% neutral-buffered formalin for 48 hours, immediately upon removal. The tissue samples were processed as routine protocol of dehydrated in ascending grades of ethanol (50\%, 70\%, 96\%, and 100\%), cleared in xylene and then embedded in paraffin. Paraffin embedded tissue samples sectioned at $5 \mu \mathrm{m}$ thickness by rotary microtome. The slides were stained with hematoxylin \& eosin and Masson Trichrome (with aniline blue) kit (Bio-Optica, Milano, Italy) for histological examination. Finally the slides were examined and photographed by using light microscope with imaging system (DM2500/DFC295; Leica, Wetzlar, Germany). The histopathological findings and classifications of nerve tissue injury examined by using light microscopy and were scored according to the classification of nerve injury by Seddon ${ }^{25)}$ and Sunderland ${ }^{27)}$ and were assessed in a blinded analysis technique by a pathologist (Table 1). The classifications of sciatic nerve tissue injury were determined and scored by microscopically examination of neurapraxia, axonotmesis and neurotmesis in the distal segment of the injured sciatic nerve tissue area with 20 and $40 \times$ objectives.

Walking track analyses were performed before the sacrification using stamp and paper method. The lengths of the third toe to heel (PL), first to fifth toe (TS), and second toe to fourth toe (IT) were measured on experimental (E) and normal sides $(\mathrm{N})$ and sciatic function index (SFI) was calculated by using the following formula :

$$
\begin{aligned}
\mathrm{SFI}= & -38.3 \times(\mathrm{EPL}-\mathrm{NPL}) / \mathrm{NPL}+109.5 \times(\mathrm{ETS}-\mathrm{NTS}) / \\
& \mathrm{NTS}+13.3 \times(\mathrm{EIT}-\mathrm{NIT}) / \mathrm{NIT}-8.8
\end{aligned}
$$

SFI score 0 represents normal nerve function and -100 total dysfunction ${ }^{20,23)}$.

All data were analysed using the SPSS ver. 20.0 software package (SPSS Inc., Chicago, IL, USA). The Mann-Whitney U test was used to compare the descriptive statistical measurements (mean, standard deviation, minimum and maximum) and the two groups of parameters that did not show a normal distribution in the quantitative data comparison. One-way analysis of variance was applied to assess the differences among the groups. Significance was evaluated at $p<0.05$.

\section{RESULTS}

The PNI and SFI scores and the serum GPX, SOD, catalase, and MDA levels are shown in Table 1. PNI score was similar in group II and group IV, and PNI score in group III was lower than group II and group IV and the difference was statistically significant $(p<0.05)$.

The MDA levels, which is also indicator of tissue damage, were parallel to the PNI score. It was minimum in group I and maximum in group II and there is a statistically significant difference between group III and all other groups $(p<0.05)$.

Table 1. The classification of nerve injury by Seddon and Sunderland

\begin{tabular}{llll}
\hline Seddon & \multicolumn{1}{c}{ Sunderland } & \multicolumn{1}{c}{ Process } & Score \\
\hline Neurapraxia & First degree & Segmental demyelination & 1 \\
Axonotmesis & Second degree & Axon severed but endoneurium intact (optimal circumstances for regeneration) & 2 \\
Axonotmesis & Third degree & $\begin{array}{c}\text { Axon discontinuity, endoneurial tube discontinuity, perineurium and fascicular } \\
\text { arrangement preserved }\end{array}$ & 3 \\
Axonotmesis & Fourth degree & $\begin{array}{c}\text { Loss of continuity of axons, endoneurial tubes, perineurium and fasciculi; epineurium } \\
\text { intact (neuroma in continuity) }\end{array}$ \\
Neurotmesis & Fifth degree & Loss of continuity of entire nerve trunk & 4 \\
\hline
\end{tabular}


All of the antioxidant enzyme levels, catalase, GPX and SOD, were also parallel to the each other. They were higher in group III than all other groups and, group II and group IV have similar results. The differences were statistically signifi-
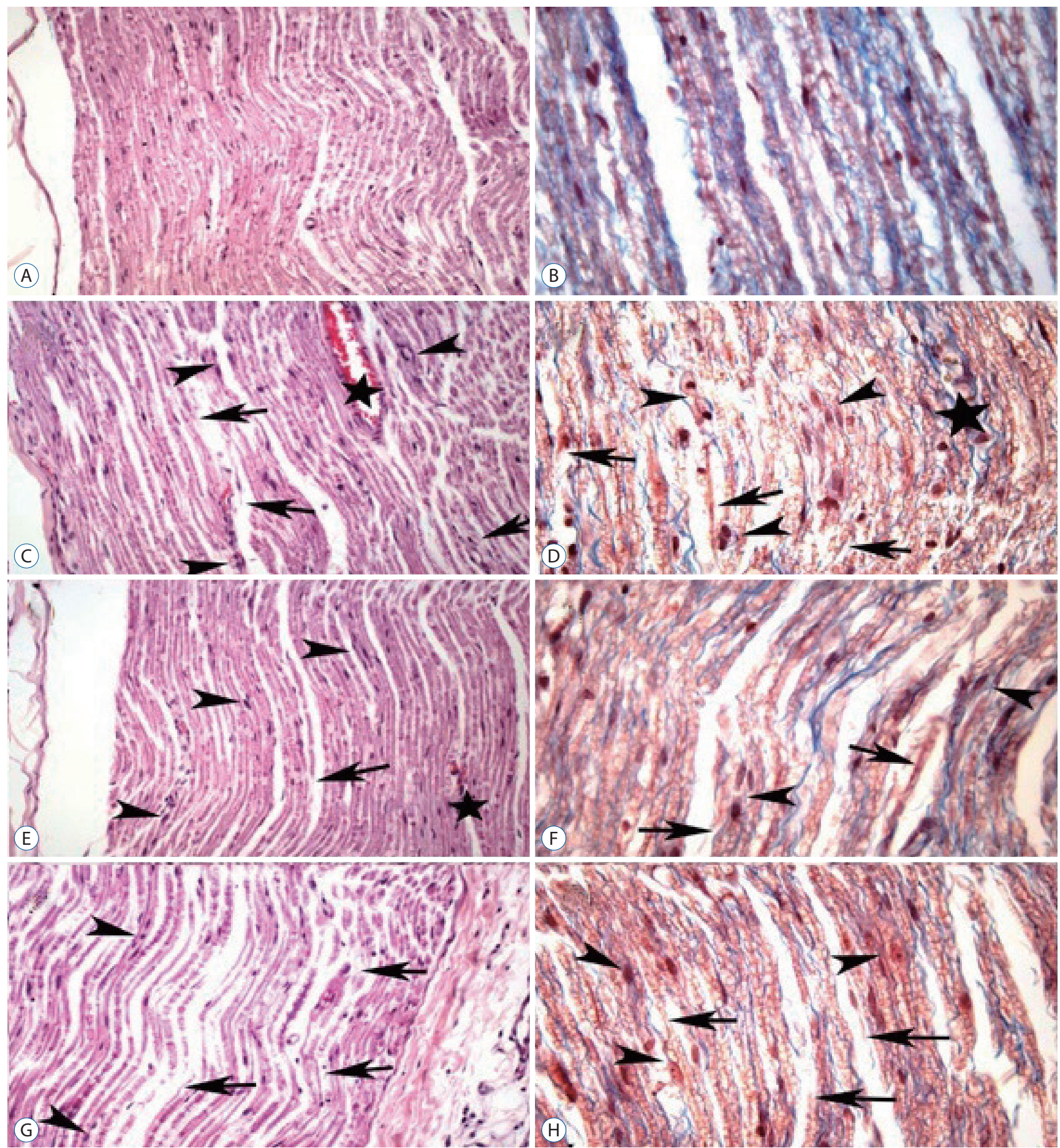

Fig. 2. Histopathology of sciatic nerve tissues; H\&E staining with $20 \times(A, C, E$, and $G)$ and $M T$ staining with $40 \times$ magnification $(B, D, F$, and $H)$. A and $B$ : Normal histological structure of sciatic nerve in group I. C and D : Proliferated and activated Schwann cells (arrowheads), segmental demyelinated nerve fibers (arrows) in group II. Proliferated and activated Schwann cells (arrowheads), axonal demyelination, axonal and endoneurial discontinuity (arrows), congestion of blood vessel in perineurium (star) in group III ( $E$ and F) and in group IV ( $G$ and $H$ ). 
cant between group III and all other groups $(p<0.05)$.

The SFI score, which is a clinical assessment, was lower in group I than the other groups and similar in groups II, III, and IV $(p>0.05)$.

When we look at the histopathological results, in group I (sham); in the evaluated sciatic nerve tissues, no significant histopathological differences were noted and the sciatic nerve tissues were showed normal histological structure (Fig. 2A and B). It has been observed that In the group II, common lesions in the distal segment of injured sciatic nerve tissues are as the following; diffuse and severe spongious appearance (axonal demyelination), axons and endoneurial tubes discontinuity, perineurium and fascicular arrangement preserved (axonotmesis-third degree) was monitored in nerve fibrils, and congestion of blood vessels in perineurium and epineurium. Diffuse Schwann cell proliferation which digested myelin, infiltration and increasing of phagocytic cells in the perineurium and between the nerve fibers were seen. Increase of the number of Schmidt-Lanterman incisures was remarkable in the some myelin sheaths (Fig. 2C and D). Axonotmesis (2nd and 3rd degree) findings related to peripheral nerve injury were also detected in the group-III (Fig. 2E and F) and group IV (Fig. 2G and $\mathrm{H}$ ) and these findings were similar to the findings in the group II, except the detected finding that regenerative changes (inflammatory cell infiltration in epineurium) and Schwann cell activation in group-III were observed more intense. The histopathological changes detected in the sciatic nerve scored according to the groups are given in the Table 2.

\section{DISCUSSION}

In the current study, we had similar results to our previous study (lacosamide treatment after spinal cord injury) ${ }^{8)}$. Histopathological results represented that there is lower damage in group III which was treated with lacosamide when compared with the others. Furthermore, the lipid peroxidation endproduct MDA levels were lowest and the antioxidant enzymes catalase, SOD, and GPX levels were highest as a result of lacosamide treatment which also explain the reason of low damage. Different from the study on spinal cord injury, there is a clinical evaluation in the current study which showed similar results in all groups.

Peripheral nerve injury may results from compression, focal contusion, traction or transection of the nerve. After first trauma, it is followed by ischemic injury, inflammation, oxidative stress and then nerve injury occurs ${ }^{10)}$. Free oxygen radicals which are produced during this period reponsible from oxidative stress and lipid peroxidation so responsible from tissue injury. Therfore, prevention of inflammation and protection against free radical-mediated oxidative stress and lipid peroxidation are the main therapeutic strategies for the treatment. For example, the neuroprotective effect of methylprednisolone, which is a reference drug for the treatment of PNI, depends on its scavenger role for free oxygen radicals and its ability to inhibit lipid peroxidation and inflammation.

The balance between oxidants and antioxidants is critical to maintain normal cellular functions. If the oxidative stress outweighs the cellular antioxidant activity, it results in tissue injury $^{30)}$. GPX, SOD, and catalase are some of the antioxidant enzymes that protect against oxidative stress by inhibiting the production of free oxygen radicals. SODs catalyse the breakdown of the superoxide anion into oxygen and hydrogen per-

Table 2. Biochemical result and pathological and clinical scores relevant to the groups

\begin{tabular}{lcccc}
\hline & Group 1 (sham) & Group 2 (PNI) & Group 3 (PNI+L) & Group 4 (PNI+PS) \\
\hline MDA (ng/mL) & $1.09 \pm 0.02^{*}$ & $1.24 \pm 0.03^{\dagger}$ & $1.14 \pm 0.04^{*, \neq \neq}$ & $1.26 \pm 0.02^{\ddagger}$ \\
GPX (ng/mL) & $0.68 \pm 0.04^{*}$ & $0.75 \pm 0.04^{\dagger}$ & $0.87 \pm 0.03^{*, \neq}$ & $0.73 \pm 0.06^{\ddagger}$ \\
SOD $(\mathrm{ng} / \mathrm{mL})$ & $0.19 \pm 0.02^{*}$ & $0.20 \pm 0.03^{\dagger}$ & $0.33 \pm 0.02^{*+, \neq}$ & $0.22 \pm 0.03^{\ddagger}$ \\
Catalase $(\mathrm{ng} / \mathrm{mL})$ & $0.4 \pm 0.03^{*}$ & $0.61 \pm 0.02^{\dagger}$ & $0.70 \pm 0.03^{*+, \neq}$ & $0.58 \pm 0.02^{\ddagger}$ \\
Nerve injury score & $0^{*}$ & $2.86 \pm 0.14^{\dagger}$ & $2.17 \pm 0.16^{*+, \neq}$ & $2.43 \pm 0.20^{\ddagger}$ \\
SFI & $-9.2576^{*}$ & -75.3961 & $-78.9625^{*}$ & -76.5683 \\
\hline
\end{tabular}

One-way ANOVA test. ${ }^{*}$ Group I vs. III ( $\left.p<0.05\right) .{ }^{\dagger}$ Group II vs. III ( $\left.p<0.05\right) .{ }^{\ddagger}$ Group III vs. IV ( $\left.p<0.05\right)$. PNI : peripheral nerve injury, L : lacosamide, PS : physiological saline, MDA : malondialdehyde, GPX : glutathione peroxidase, SOD : superoxide dismutase, SFI : sciatic functional index 
oxide. GPX reduces hydrogen peroxide and lipid peroxide to water and lipid alcohols. Catalases are also enzymes that catalyse the conversion of hydrogen peroxide to water and oxygen. If the activity of these enzymes is decreased, the amount of free oxygen radicals increases or vice versa. So, after trauma, these enzymes are expected to increase to protect the body. Choi et al. ${ }^{6}$ reported increases of catalase and GPX expression after lacosamide pretreatment in experimentally induced transient cerebral ischaemia in gerbils and emphasised the neuroprotective effect by this increased antioxidant activity. In current study, there was also an increase in GPX, SOD and catalase in group III (Table 2). These results indicate the neuroprotective effect of lacosamide resulting from increased antioxidant enzyme activity.

Lipid peroxidation is another crucial step in the tissue injury after trauma ${ }^{13,15)}$. Free oxygen radicals damage membrane lipids and alter membrane fluidity and permeability which results in cellular dysfunction ${ }^{30)}$. MDA is the final product of these reactions and widely used as a marker of tissue injury. Solmaz et al. reported the potential lipid peroxidation-inhibiting effect of lacosamide on rats in a study on sepsis-induced critical illness polyneuropathy ${ }^{28)}$. Although sepsis and trauma are two different conditions, lipid peroxidation plays an important role in the ethiopathogenesis of both. Therefore, we suggest that lacosamide, which reduces lipid peroxidation, also has a protective effect in SCI. Consequently, the decrease in MDA serum levels was an expected result in group III and supports our hypothesis of a neuroprotective effect of lacosamide in PNI.

In clinical assessment according to the walking track analysis in groups II, III, and IV, all rats had similar SFI scores. There was no effect of lacozamide in functional recovery. We think that one week follow-up, which can be a limitation for this study, may not be enough for the assesment of clinical effectiveness. Ozbek et al. ${ }^{18)}$ observed significant motor improvement at 4 weeks after treatment after experimental peripheral nerve injury in rats.

\section{CONCLUSION}

In conclusion, this is the first study to investigate the neuroprotective effects of lacosamide after PNI. The biochemical and histological analyses revealed that lacosamide has neuro- protective effect and may be useful in the treatment of PNI. However, studies with long follow-up are needed to clinical assessment.

\section{CONFLICTS OF INTEREST}

No potential conflict of interest relevant to this article was reported.

\section{AUTHOR CONTRIBUTIONS}

\author{
Conceptualization : SD \\ Data curation : SD, KU \\ Formal analysis : ASB, AU \\ Funding acquisition : SD, SE \\ Methodology : SD, KU \\ Project administration : SD, SE \\ Visualization : YA, AC \\ Writing - original draft : SD, KU \\ Writing - review \& editing : SD
}

\section{ORCID}

$\begin{array}{ll}\text { Serdar Demiroz } & \text { https://orcid.org/0000-0002-2403-3750 } \\ \text { Koray Ur } & \text { https://orcid.org/0000-0002-2755-5992 } \\ \text { Aydin Sukru Bengu https://orcid.org/0000-0002-7635-4855 } \\ \text { Aykut Ulucan } & \text { https://orcid.org/0000-0001-8844-8237 } \\ \text { Yunus Atici } & \text { https://orcid.org/0000-0002-9661-4618 } \\ \text { Sinan Erdogan } & \text { https://orcid.org/0000-0002-8517-3925 } \\ \text { Alper Cirakli } & \text { https://orcid.org/0000-0002-9879-312X } \\ \text { Sevki Erdem } & \text { https://orcid.org/0000-0002-3977-3380 }\end{array}$

\section{References}

1. Ahn JY, Yan BC, Park JH, Ahn JH, Lee DH, Kim IH, et al. : Novel antiepileptic drug lacosamide exerts neuroprotective effects by decreasing glial activation in the hippocampus of a gerbil model of ischemic stroke. Exp Ther Med $10:$ 2007-2014, 2015

2. Altun I, Çiralik H : Histopathological effects of tissue adhesives on experimental peripheral nerve transection model in rats. J Korean Neurosurg Soc 58 : 504-507, 2015 
3. Baek WK, Jang BC, Lim JH, Kwon TK, Lee HY, Cho CH, et al. : Inhibitory modulation of ATP-sensitive potassium channels by gallateester moiety of (-)-epigallocatechin-3-gallate. Biochem Pharmacol 70 : 15601567, 2005

4. Bastianetto S, Yao ZX, Papadopoulos V, Quirion R : Neuroprotective effects of green and black teas and their catechin gallate esters against beta-amyloid induced toxicity. Eur J Neurosci 23 : 55-64, 2006

5. Brown JE, Khodr H, Hider RC, Rice-Evans CA : Structural dependence of flavonoid interactions with $\mathrm{Cu} 2+$ ions: implications for their antioxidant properties. Biochem J 330 (Pt 3) : 1173-1178, 1998

6. Choi HY, Park JH, Chen BH, Shin BN, Lee YL, Kim IH, et al. : Increases of catalase and glutathione peroxidase expressions by lacosamide pretreatment contributes to neuroprotection against experimentally induced transient cerebral ischemia. Neurochem Res 41 : 2380-2390, 2016

7. Cui SS, Yang CP, Bowen RC, Bai O, Li XM, Jiang W, et al. : Valproic acid enhances axonal regeneration and recovery of motor function after sciatic nerve axotomy in adult rats. Brain Res 975 : 229-236, 2003

8. Demiroz S, Ur K, Ulucan A, Bengu AS, Ur FD, Gergin 00, et al. : Neuroprotective effects of lacosamide in experimental traumatic spinal cord injury in rats. Turk Neurosurg 29 : 718-723, 2019

9. Fenrich $\mathrm{K}$, Gordon $\mathrm{T}$ : Canadian Association of Neuroscience review: axonal regeneration in the peripheral and central nervous systems-current issues and advances. Can J Neurol Sci 31 : 142-156, 2014

10. Ghayour MB, Abdolmaleki A, Behnam-Rassouli M : The effect of Riluzole on functional recovery of locomotion in the rat sciatic nerve crush model. Eur J Trauma Emerg Surg 43 : 691-699, 2017

11. Gülsen I, Ak H, Kara M, Gökalp A, Akyol V, Koçak ÖF, et al. : The acute effects of thymoquinone on acute peripheral nerve injury: an experimental study. Ulus Travma Acil Cerrahi Derg 22 : 526-530, 2016

12. Gutteridge JM : Lipid peroxidation and antioxidants as biomarkers of tissue damage. Clin Chem 41(12 Pt 2) : 1819-1828, 1995

13. Halliwell B, Gutteridge JM : Oxidative stress in Free Radicals in Biology and Medicine, 3rd ed. New York : Oxford University Press, 1999, pp246-350

14. Kim SH, Park BN, Kim SW : The effect of phosphodiesterase-4-specific inhibitor in the rat model of spinal nerve ligation. J Korean Neurosurg Soc 50 : 109-113, 2011

15. Koc RK, Akdemir H, Karakücük El, Oktem IS, Menkü A : Effect of methylprednisolone, tirilazad mesylate and vitamin $\mathrm{E}$ on lipid peroxidation after experimental spinal cord injury. Spinal Cord 37 : 29-32, 1999

16. Lundborg $G$ : Intraneural microcirculation. Orthop Clin North Am 19 : 1-12, 1998

17. Marques SA, Garcez VF, Del Bel EA, Martinez AM : A simple, inexpen- sive and easily reproducible model of spinal cord injury in mice: morphological and functional assessment. J Neurosci Methods 177 : 183193, 2009

18. Ozbek Z, Aydin HE, Kocman AE, Ozkara E, Sahin E, Bektur E, et al. : Neuroprotective effect of genistein in peripheral nerve injury. Turk Neurosurg $27: 816-822,2017$

19. Özer $H$ : The effects poli (3-hidroksibütirat) (PHB) and chitosan modificated tubular graft and mezenchymal stem cell on axonal regeneration in experimental sciatic nerve injury. Ankara : Hacettepe Üniversitesi, 2013

20. Ozmen S, Ayhan S, Latifoglu O, Siemionow M : Stamp and paper method: a superior technique for the walking track analysis. Plast Reconstr Surg $109:$ 1760-1761, 2002

21. Panaite PA, Barakat-Walter I: Thyroid hormone enhances transected axonal regeneration and muscle reinnervation following rat sciatic nerve injury. J Neurosci Res 88 : 1751-1763, 2010

22. Pitkänen A, Immonen $R$, Ndode-Ekane $X$, Gröhn 0 , Stöhr T, Nissinen J : Effect of lacosamide on structural damage and functional recovery after traumatic brain injury in rats. Epilepsy Res 108 : 653-665, 2014

23. Sarikcioglu $L$, Demirel BM, Utuk $A$ : Walking track analysis: an assessment method for functional recovery after sciatic nerve injury in the rat. Folia Morphol (Warsz) 68 : 1-7, 2009

24. Schenker M, Riederer BM, Kuntzer T, Barakat-Walter I: Thyroid hormones stimulate expression and modification of cytoskeletal protein during rat sciatic nerve regeneration. Brain Res 957 : 259-270, 2002

25. Seddon HJ : Three types of nerve injury. Brain $66: 237-288,1943$

26. Shir Y, Campbell JN, Raja SN, Seltzer Z : The correlation between dietary soy phytoestrogens and neuropathic pain behavior in rats after partial denervation. Anesth Analg 94 : 421-426, 2002

27. Sunderland $S$ : The anatomy and physiology of nerve injury. Muscle Nerve $13: 771-784,1990$

28. Tator $\mathrm{CH}$, Fehlings $\mathrm{MG}$ : Review of the secondary injury theory of acute spinal cord trauma with emphasis on vascular mechanisms. J Neurosurg $75: 15-26,1991$

29. Wu F, Xing D, Peng Z, Rao $T$ : Enhanced rat sciatic nerve regeneration through silicon tubes implanted with valproic acid. J Reconstr Microsurg $24: 267-276,2008$

30. Yang YH, Wang Z, Zheng J, Wang R : Protective effects of gallic acid against spinal cord injury-induced oxidative stress. Mol Med Rep 12 : 3017-3024, 2015

31. Zochodne DW, Ho LT : Endoneurial microenvironment and acute nerve crush injury in the rat sciatic nerve. Brain Res 535 : 43-48, 1990 